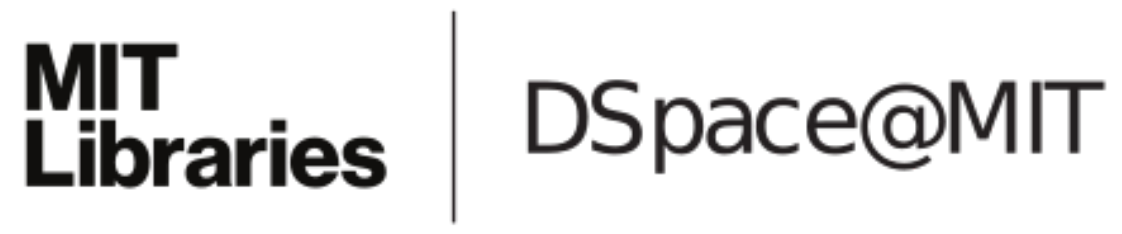

\author{
MIT Open Access Articles
}

Design of Stable Nanocrystalline Alloys

The MIT Faculty has made this article openly available. Please share how this access benefits you. Your story matters.

Citation: Chookajorn, T., H. A. Murdoch, and C. A. Schuh. “Design of Stable Nanocrystalline Alloys." Science 337, no. 6097 (August 23, 2012): 951-954.

As Published: http://dx.doi.org/10.1126/science.1224737

Publisher: American Association for the Advancement of Science (AAAS)

Persistent URL: http://hdl.handle.net/1721.1/80308

Version: Author's final manuscript: final author's manuscript post peer review, without publisher's formatting or copy editing

Terms of Use: Article is made available in accordance with the publisher's policy and may be subject to US copyright law. Please refer to the publisher's site for terms of use. 


\title{
Title: Design of Stable Nanocrystalline Alloys
}

\author{
Authors: Tongjai Chookajorn ${ }^{1}$, Heather A. Murdoch ${ }^{1}$, Christopher A. Schuh ${ }^{1}$ *
}

\author{
Affiliations: \\ ${ }^{1}$ Department of Materials Science and Engineering, MIT, Cambridge, MA 02139, USA. \\ *Correspondence to: schuh@mit.edu
}

\begin{abstract}
:
Nanostructured metals are generally unstable; their grains grow rapidly even at low temperatures, rendering them difficult to process and often unsuitable for usage. Alloying has been found to improve stability, but only in a few empirically discovered systems. We have developed a theoretical framework with which stable nanostructured alloys can be designed. A nanostructure stability map based on a thermodynamic model is applied to design stable nanostructured tungsten alloys. We identify a candidate alloy, W-Ti, and demonstrate substantially enhanced stability for the high-temperature, long-duration conditions amenable to powder-route production of bulk nanostructured tungsten. This nanostructured alloy adopts a heterogeneous chemical distribution that is anticipated by the present theoretical framework, but unexpected based on conventional bulk thermodynamics.
\end{abstract}

\section{Main Text:}

When the grain, or crystallite, size in a polycrystal is reduced below about $100 \mathrm{~nm}$, grain boundary or interface physics begin to dominate over conventional crystal physics (1-4). A variety of interesting and useful properties emerge in such nanostructured materials (5-10), but their large volume fraction of grain boundaries also comes with an inherent instability. Many nanocrystalline materials of interest for both fundamental study and engineering application are now recognized as significantly out of equilibrium and undergo rapid evolution to coarser structures even at modest temperatures (11). Such coarsening tendencies impede the use of these materials at elevated temperatures, as well as the development of scalable processing routes, which would commonly involve at least one substantial thermal excursion, for example, to sinter processed powders into a dense nanocrystalline compact.

Over the past few years, attention has shifted to the problem of stabilizing nanocrystalline structures by alloying. Whereas one approach to the problem is a classical kinetic strategy, that is, including alloying elements to slow grain growth, there is increasing interest in the notion of genuine thermodynamic stabilization of grain boundaries (12-18). In analogy to microemulsions, in which the addition of surfactant is used to stabilize interfacial area (19), the concept of thermodynamic nanostructure stabilization is to add to a polycrystal an alloying element (solute) selected for its preference to occupy grain boundary sites vis-à-vis those in the crystal interiors to relieve the energy penalty of the interfaces. The grain boundary energy, $\gamma$, is lowered from that of a pure material, $\gamma_{0}$, through such segregation, which in a simplified and linearized view can be written (12)

$\gamma=\gamma_{0}-\Gamma\left(\Delta H^{\mathrm{seg}}+\mathrm{k} T \ln X\right)$ 
where the specific solute excess at the boundary, $\Gamma$, lowers the enthalpy by $\Delta H^{\text {seg }}$, the enthalpy of segregation, and raises the entropy via $\mathrm{k} T \ln X$, with $\mathrm{k} T$ the thermal energy and $X$ the composition.

A few systems, both simulated and experimental, have provided evidence that this thermodynamic approach can suppress grain growth and stabilize nanostructured polycrystals (16, 20-28). Some authors have provided guidelines for estimating the grain boundary segregation strength given a base element by identifying preferred features of the solute, for example, atomic size mismatch with the solvent (16) or low bulk solid solubility $(23,24)$, both of which are presumed to correlate with a higher tendency for solute rejection into grain boundaries. By and large, these approaches amount to semi-empirical preferences for alloy systems that might exhibit grain boundary segregation and do not generally speak to true thermodynamic stability of nanostructures, which requires consideration of the relative stability of nanostructured phases to competing bulk phases. For example, existing approaches often suggest systems that experience other problematic structural instabilities beyond just normal grain growth, that is, abnormal grain growth or phase decomposition (21-24). We advance a thermodynamic model, with which nanostructure stability maps can be generated and used as a design tool. One newly predicted system, namely $\mathrm{W}$ with a minority addition of $\mathrm{Ti}$, is evaluated and demonstrates stability at a length scale of around $20 \mathrm{~nm}$ over long durations at elevated temperatures.

To assess the efficacy of solutes in stabilizing nanostructures, we describe the mixing free energy of a nanostructured binary system with separate energetic interactions in grain and intergranular regions. The two regions are not treated as separate phases, per se, but are geometrically connected to one another such that a reduction in grain size, $d$, causes an increase in the grain boundary volume fraction, $f_{\mathrm{gb}}$, which follows the scaling $f_{\mathrm{gb}}=1-\left(\frac{\mathrm{d}-\mathrm{t}}{\mathrm{d}}\right)^{3}$, where $t$ is the mean grain boundary thickness and $d \geq t$. The model, presented in a preliminary form by Trelewicz and Schuh (29), reduces to a classical regular solution model in the limit of infinite grain size and also reproduces a grain boundary energy expression like that of Eq. (1) in the proper limit. The model thus essentially provides the form of Gibbs free energy surfaces for mixing, $\Delta G^{\text {mix }}$, as a function of both $f_{\mathrm{gb}}$ and $X$ as

$\Delta G^{\mathrm{mix}}=\left(1-f_{\mathrm{gb}}\right) \Delta G_{\mathrm{c}}^{\mathrm{mix}}+f_{\mathrm{gb}} \Delta G_{\mathrm{gb}}^{\mathrm{mix}}+z v f_{\mathrm{gb}}\left(X_{\mathrm{gb}}-X_{\mathrm{c}}\right)\left[\left(2 X_{\mathrm{gb}}-1\right) \omega_{\mathrm{gb}}-\frac{1}{z t}\left(\Omega^{\mathrm{B}} \gamma^{\mathrm{B}}-\Omega^{\mathrm{A}} \gamma^{\mathrm{A}}\right)\right]$

where the subscripts denote the two regions, crystal (c) and grain boundary (gb), and the superscripts denote the two alloy components, A (solvent) and B (solute). The symbol $\Delta G^{\text {mix }}$ denotes the Gibbs free energy for a regular solution model, written for the subscripted components: the first two terms in the equation thus amount to a weighted average of two regular solutions, one for the crystals and one for the grain boundary regions. The additional terms are associated with the geometrical way in which those two regions interact. The bond energies are collected in the usual way into an interaction parameter, $\omega$, of which there are two (crystal and grain boundary); additional terms include the coordination number, $z$; transition bond fraction, $v$; 
and atomic volume, $\Omega$. The most important point for this model is that it describes a free energy surface in composition-grain size space. We are interested in finding alloys where there are global minima in such a surface, that is, where there is a thermodynamically preferred grain size dictated by the grain boundary segregation state at a given composition.

For the purposes of developing a design approach, it is useful to focus on two key thermodynamic parameters, which together contain all of the most relevant physics of the problem: the enthalpy of mixing in the crystalline state, $\Delta H^{\mathrm{mix}}=z \omega_{\mathrm{c}} X(1-X)$, to represent the grain interior, and the dilute-limit enthalpy of segregation, $\Delta H^{\mathrm{seg}}=z\left[\omega_{\mathrm{c}}-\frac{\omega_{\mathrm{gb}}}{2}-\frac{\left(\Omega^{\mathrm{B}} \gamma^{\mathrm{B}}-\Omega^{\mathrm{A}} \gamma^{\mathrm{A}}\right)}{2 z t}\right]$, to capture the thermodynamics of the grain boundary environment, which incorporates chemical interactions, elastic mismatch, and the mismatch in interfacial energies. These two parameters form the axes of a nanostructure stability map, as shown in Fig. 1A. By fixing other quantities (most notably, temperature), we can iterate the values of these two parameters over physically plausible ranges and calculate the shape of the free energy surface given by Eq. (2). We identify the global minima that correspond to nanocrystalline grain sizes with a particular grain boundary segregation profile. These minima are then compared to the energies of other possible bulk states. Combinations of parameters that have stable nanocrystalline states are marked by green points; those without, red x's. The conditions under which a nanocrystalline system would be stable or not are thus demarcated by the green and red regions of Fig. 1A.

Examples of how nanocrystalline states are evaluated for stability with respect to bulk structures are shown in Fig. 1, B and C, for conditions in which the nanocrystalline structure is stable and unstable, respectively. In these panels, the blue curves represent local cuts of the free energy surface of Eq. (2) and the lowest-energy state available for particular nanocrystalline grain sizes and segregation states. There are many such states across a range of composition, as reflected by the multiple distinct curves shown in the panels. The black curves are the bulk regular solution; the systems shown exhibit a miscibility gap denoted by the common-tangent dashed lines because they have positive $\Delta H^{\text {mix }}$. The difference between Fig. 1B and 1C lies in the position of the nanostructure free energy curves with respect to the bulk phase separation common-tangent line. In Fig. 1B, the nanostructured states are stable, that is, have lower Gibbs free energy than the competing bulk phase separated state. In Fig. 1C, there are nanostructured states that could exist, but these are less stable than bulk phase separation. The difference between the two systems in Fig. 1, B and C is fundamental, and we specifically seek to identify alloys that fall into the first category rather than the second.

To demonstrate the utility of the map in Fig. 1A, we consider one problem in nanostructured alloy design, namely the development of nanocrystalline tungsten, which is of interest for its anticipated high strength and unique capacity for shear localization $(30,31)$, but which has proven challenging to produce in bulk form because of its extremely high melting (and therefore processing) temperature. The finest grain sizes reported in a bulk tungsten material are about $40 \mathrm{~nm}$, and this required a complex processing route involving multiple severe 
deformation steps (32). In principle, tungsten could be made in bulk form through a powder route, but the requirement of high-temperature sintering is usually a debilitating roadblock to such routes because of nanostructure coarsening; tungsten can generally only be sintered at temperatures above about $1050^{\circ} \mathrm{C}$ even with sintering aids $(33,34)$.

We use Eq. (2) and Fig. 1A to develop a stable nanostructured $\mathrm{W}$ alloy by first placing particular alloying elements for $\mathrm{W}$ on the map. This requires estimates of both $\Delta H^{\text {mix }}$ and $\Delta H^{\text {seg }}$ for each possible binary system. We have calculated these quantities and populated Fig. 1A with all of the alloys (35) that exhibit positive $\Delta H^{\text {mix }}$ with $\mathrm{W}$ and for which reliable data are available (values listed in table S1, with more details on the calculations provided in the supplementary materials). There is, of course, some uncertainty in all of these estimates, generally of the magnitude illustrated for one of the represented alloys, W-Ti, on the map.

Fig. 1A identifies a variety of candidate stable nanostructured materials that lie in the green region. Closer consideration reveals some unexpected predictions. For example, the likelihood of nanostructure stability has often been estimated by solute segregation strength based on atomic size mismatch and/or solubility. However, in the present system, the solute with the highest size difference listed in table $\mathrm{S} 1$ ( $\mathrm{Sr}, \sim 55 \%$ mismatch with $\mathrm{W}$ ) and those with the lowest solubility (Ag and $\mathrm{Cu}$, essentially zero solubility) lie decidedly in the red bulk stable region in Fig. 1A. Also counterintuitively, the element with the lowest value of $\Delta H^{\text {seg }}$ in the set, $\mathrm{Ti}$, is actually one of the most suitable candidates, being safely within the nanocrystalline stable region. In fact, Ti would be counterindicated by conventional approaches, which would seek low bulk solubility [Ti has extremely high solubility in $\mathrm{W}$ of 48 atomic $\%$ at $1100^{\circ} \mathrm{C}(36)$ ] and high atomic size mismatch ( $\mathrm{Ti}$ has a low-to-moderate mismatch with $\mathrm{W}$ of only $\sim 6 \%$ ).

On the basis of these results, we produced a W-20 atomic \% Ti alloy with an average grain size around $20 \mathrm{~nm}$ (Fig. 2B) by high-energy ball milling (35); the output of this process is microscale powders, where each particle comprises many nanocrystalline grains. As a control, we also produced unalloyed nanocrystalline $\mathrm{W}$ with about the same grain size through the same process. The powders were then equilibrated at $1100^{\circ} \mathrm{C}$ in an argon atmosphere for one week; at this temperature the dominant diffusion pathway is intergranular, and the mean diffusion distance is several micrometers, which is thousands of times greater than the grain size. Pre- and postannealing structures were characterized to explore the stability of the nanostructure, and Fig. 2 shows the most important results of such characterizations.

After one week at $1100^{\circ} \mathrm{C}$, the unalloyed nanocrystalline $\mathrm{W}$ exhibits the typical instability of such materials, with grain coarsening to the micrometer scale, as shown in Fig. $2 \mathrm{C}$. On the other hand, the $\mathrm{W}-20$ atomic \% Ti alloy retains a uniform nanostructure with a nominally unchanged average grain size of about $20 \mathrm{~nm}$. This stability can be seen visually in Fig. 2D and quantitatively in the grain size distributions of Fig. 2A. With Ti present, the system adopts a complex alloy configuration where Ti and $\mathrm{W}$ are heterogeneously distributed on the nanoscale as a polycrystalline body-centered cubic (BCC) structure, with no signatures of any amorphous 
content. This heterogeneous distribution is illustrated by the chemical arrangement in the equilibrated alloy in Fig. 3, with Fig. 3A showing the atomic contrast between W and Ti and Fig. 3B showing a local chemical map based on energy dispersive spectroscopy (35). A compositional line scan in Fig. 3C reveals the magnitude of the Ti composition ranging from near 0 atomic $\%$ to about 50 atomic $\%$.

The nanoscale chemical distribution seen in Fig. 3 is not expected for a bulk equilibrium alloy, where $\mathrm{Ti}$ is soluble to 48 at.\% in $\mathrm{W}$ at the equilibration temperature, and a homogeneous chemical distribution should be observed. This solute distribution is a consequence of the nanostructure: the high volume fraction of grain boundaries creates different chemical configurations, and a lower energy state results from the heterogeneous solute distribution. In a nanoscale structure, a heterogeneous solute distribution is explicitly expected from Eq. (2).

From a technological standpoint, the results in Figs. 2 and 3 suggest that nanocrystalline tungsten can, in principle, be made sufficiently stable to survive a typical consolidation thermal cycle. Given the exceptionally high strength of nanocrystalline BCC metals (37) and the unusual secondary properties (such as shear localization) that emerge at these grain sizes, the present results may speak to a new family of engineering tungsten alloys. At the same time, our experimental work on $\mathrm{W}-\mathrm{Ti}$ is simply an example of a single alloy design exercise; the above approach may be applied again to a number of different base metals.

\section{References and Notes:}

1. K. Lu, L. Lu, S. Suresh, Strengthening Materials by Engineering Coherent Internal Boundaries at the Nanoscale. Science 324, 349 (April 17, 2009, 2009).

2. J. R. Weertman et al., Structure and mechanical behavior of bulk nanocrystalline materials. MRS Bulletin 24, 44 (1999).

3. H. Van Swygenhoven, J. R. Weertman, Deformation in nanocrystalline metals. Materials Today 9, 24 (2006).

4. X. Z. Liao, F. Zhou, E. J. Lavernia, D. W. He, Y. T. Zhu, Deformation twins in nanocrystalline A1. Applied Physics Letters 83, 5062 (2003).

5. L. Lu, M. L. Sui, K. Lu, Superplastic Extensibility of Nanocrystalline Copper at Room Temperature. Science 287, 1463 (February 25, 2000, 2000).

6. L. Lu, Y. Shen, X. Chen, L. Qian, K. Lu, Ultrahigh Strength and High Electrical Conductivity in Copper. Science 304, 422 (April 16, 2004, 2004).

7. Y. Wang, M. Chen, F. Zhou, E. Ma, High tensile ductility in a nanostructured metal. Nature 419, 912 (2002).

8. D. B. Witkin, E. J. Lavernia, Synthesis and mechanical behavior of nanostructured materials via cryomilling. Progress in Materials Science 51, 1 (2006).

9. B. Poudel et al., High-Thermoelectric Performance of Nanostructured Bismuth Antimony Telluride Bulk Alloys. Science 320, 634 (May 2, 2008, 2008).

10. M. E. McHenry, M. A. Willard, D. E. Laughlin, Amorphous and nanocrystalline materials for applications as soft magnets. Progress in Materials Science 44, 291 (1999).

11. M. Ames et al., Unraveling the nature of room temperature grain growth in nanocrystalline materials. Acta Materialia 56, 4255 (2008).

12. J. Weissmüller, Alloy effects in nanostructures. Nanostructured Materials 3, 261 (1993). 
13. R. Kirchheim, Grain coarsening inhibited by solute segregation. Acta Materialia 50, 413 (2002).

14. R. Kirchheim, Reducing grain boundary, dislocation line and vacancy formation energies by solute segregation. I. Theoretical background. Acta Materialia 55, 5129 (2007).

15. R. Kirchheim, Reducing grain boundary, dislocation line and vacancy formation energies by solute segregation: II. Experimental evidence and consequences. Acta Materialia 55, 5139 (2007).

16. P. C. Millett, R. P. Selvam, A. Saxena, Stabilizing nanocrystalline materials with dopants. Acta Materialia 55, 2329 (2007).

17. J. Luo, H. Cheng, K. M. Asl, C. J. Kiely, M. P. Harmer, The Role of a Bilayer Interfacial Phase on Liquid Metal Embrittlement. Science 333, 1730 (September 23, 2011, 2011).

18. M. Baram, D. Chatain, W. D. Kaplan, Nanometer-Thick Equilibrium Films: The Interface Between Thermodynamics and Atomistics. Science 332, 206 (April 8, 2011, 2011).

19. M. Kahlweit, Microemulsions. Science 240, 617 (April 29, 1988, 1988).

20. A. J. Detor, C. A. Schuh, Grain boundary segregation, chemical ordering and stability of nanocrystalline alloys: Atomistic computer simulations in the $\mathrm{Ni}-\mathrm{W}$ system. Acta Materialia 55, 4221 (2007).

21. A. J. Detor, C. A. Schuh, Microstructural evolution during the heat treatment of nanocrystalline alloys. Journal of Materials Research 22, 3233 (2007).

22. T. Hentschel, D. Isheim, R. Kirchheim, F. Muller, H. Kreye, Nanocrystalline Ni-3.6 at.\% $\mathrm{P}$ and its transformation sequence studied by atom-probe field-ion microscopy. Acta Materialia 48, 933 (2000).

23. P. Choi, M. da Silva, U. Klement, T. Al-Kassab, R. Kirchheim, Thermal stability of electrodeposited nanocrystalline Co-1.1at.\%P. Acta Materialia 53, 4473 (2005).

24. K. A. Darling, B. K. VanLeeuwen, C. C. Koch, R. O. Scattergood, Thermal stability of nanocrystalline $\mathrm{Fe}-\mathrm{Zr}$ alloys. Materials Science and Engineering: A 527, 3572 (2010).

25. F. Liu, R. Kirchheim, Grain boundary saturation and grain growth. Scripta Materialia 51, 521 (2004).

26. C. E. Krill, H. Ehrhardt, R. Birringer, Thermodynamic stabilization of nanocrystallinity. Zeitschrift für Metallkunde 96, 1134 (2005).

27. S. G. Mayr, D. Bedorf, Stabilization of $\mathrm{Cu}$ nanostructures by grain boundary doping with Bi: Experiment versus molecular dynamics simulation. Physical Review B 76, 024111 (2007).

28. C. Koch, R. Scattergood, K. Darling, J. Semones, Stabilization of nanocrystalline grain sizes by solute additions. Journal of Materials Science 43, 7264 (2008).

29. J. R. Trelewicz, C. A. Schuh, Grain boundary segregation and thermodynamically stable binary nanocrystalline alloys. Physical Review B 79, 094112 (2009).

30. D. Roundy, C. R. Krenn, M. L. Cohen, J. W. Morris, The ideal strength of tungsten. Philosophical Magazine A 81, 1725 (2001).

31. Q. Wei et al., Plastic flow localization in bulk tungsten with ultrafine microstructure. Applied Physics Letters 86, 101907 (2005).

32. L. J. Kecskes et al., Grain size engineering of bcc refractory metals: Top-down and bottom-up-Application to tungsten. Materials Science and Engineering: A 467, 33 (2007). 
33. H. W. Hayden, J. H. Brophy, The Activated Sintering of Tungsten with Group VIII Elements. Journal of The Electrochemical Society 110, 805 (1963).

34. R. German, Z. Munir, Enhanced low-temperature sintering of tungsten. Metallurgical and Materials Transactions A 7, 1873 (1976).

35. Materials and methods are available as supplementary material on Science Online.

36. S. Jonsson, Reevaluation of the Ti-W system and prediction of the Ti-W-N phase diagram. Zeitschrift für Metallkunde 87, 784 (1996).

37. C. R. Krenn, D. Roundy, J. W. Morris Jr, M. L. Cohen, Ideal strengths of bcc metals. Materials Science and Engineering: A 319-321, 111 (2001).

38. F. R. de Boer, R. Boom, W. C. M. Mattens, A. R. Miedema, A. K. Niessen, Cohesion in Metals: Transition Metal Alloys. (1988).

39. H. Bakker, Enthalpies in Alloys: Miedema's Semi-Empirical Model. Materials Science Foundations (Trans Tech Publications, Enfield, New Hampshire, 1998).

40. S. Kwang Lee, D. Nyung Lee, Calculation of phase diagrams using partial phase diagram data. Calphad 10, 61 (1986).

41. P. Wynblatt, D. Chatain, Anisotropy of segregation at grain boundaries and surfaces. Metallurgical and Materials Transactions A 37, 2595 (2006).

42. P. Wynblatt, Z. Shi, Relation between grain boundary segregation and grain boundary character in FCC alloys. Journal of Materials Science 40, 2765 (2005).

43. K. A. Darling et al., Stabilized nanocrystalline iron-based alloys: Guiding efforts in alloy selection. Materials Science and Engineering: A 528, 4365 (2011).

44. J. Friedel, Electronic structure of primary solid solutions in metals. Advances in Physics 3, 446 (1954).

45. G. Cliff, G. W. Lorimer, The quantitative analysis of thin specimens. Journal of Microscopy 103, 203 (1975).

46. G. W. C. Kaye, T. H. Laby, Tables of physical and chemical constants and some mathematical functions. (Longman, 1973).

47. E. Lassner, W.-D. Schubert, Tungsten: Properties, Chemistry, Technology of the Element, Alloys, and Chemical Compounds. (Springer, ed. 1, 1999).

48. H. L. Skriver, N. M. Rosengaard, Surface energy and work function of elemental metals. Physical Review B 46, 7157 (1992).

49. H. Jones, The Surface Energy of Solid Metals. Metal Science 5, 15 (1971).

50. O. N. Senkov, M. Dubois, J. J. Jonas, Elastic moduli of titanium-hydrogen alloys in the temperature range 20 degrees $\mathrm{C}$ to 1100 degrees C. Metall. Mater. Trans. A-Phys. Metall. Mater. Sci. 27, 3963 (1996).

\section{Supplementary Materials}

www.sciencemag.org

Materials and Methods

Figs. S1, S2

Tables S1, S2

References (38-50)

Acknowledgments: This work was supported by the US Army Research Office, under grant No. W911NF-09-1-0422. Additional information and data used in this work can be found in the Supplementary Materials. 

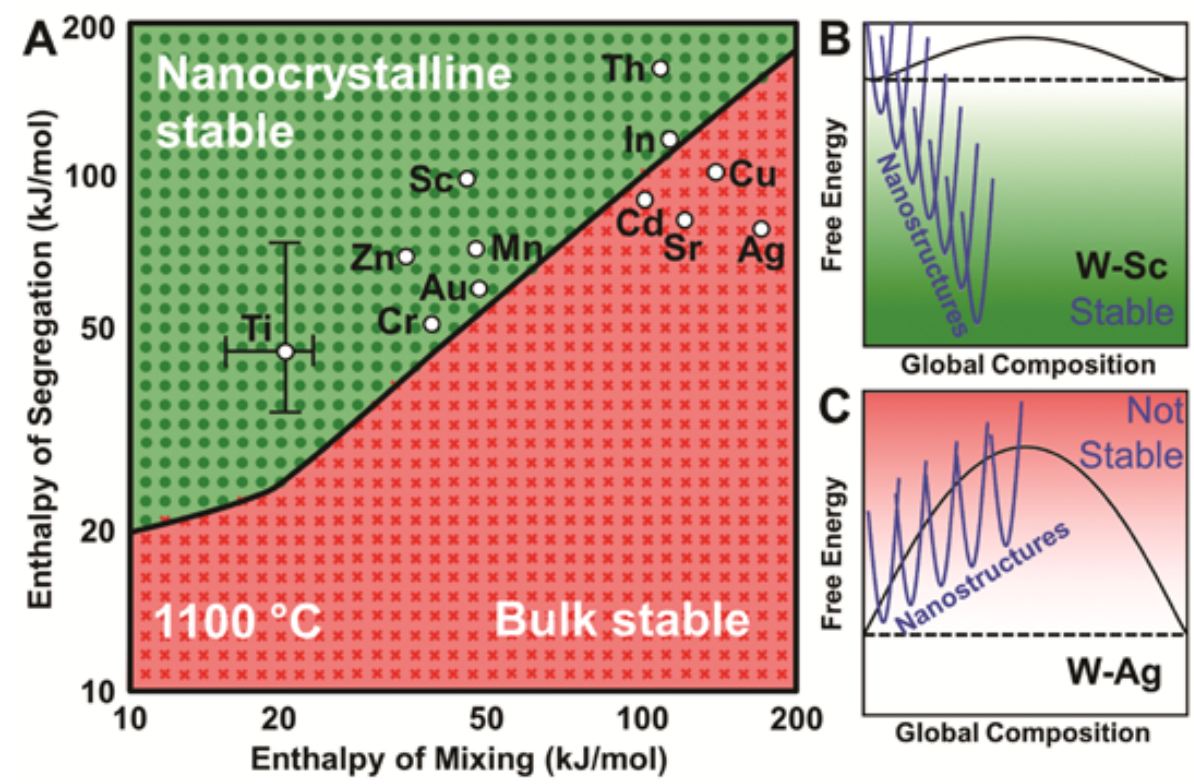

Fig. 1. The nanostructure stability map for tungsten based alloys at $1100^{\circ} \mathrm{C}$, calculated on the basis of variation of the enthalpy parameters in Eq. (2) (A). For each combination of parameters, the free energy of nanocrystalline structures is compared to that of the bulk regular solution (for details of the comparison, see Figs. S1 and S2). An example case for the nanocrystalline stable region is presented in (B), for a specific alloy of W-Sc. The free energy of the nanostructured phases is below that of the regular solution common tangent (dashed line). In (C), a bulk stable case where the nanostructured phases fall above the common tangent line is shown; the W-Ag system will then prefer to phase separate at bulk scales as dictated by the bulk regular solution thermodynamics. Particular binary tungsten alloys are placed on the map after calculating their enthalpies of mixing and segregation; for W-Ti, the typical ranges of uncertainty of these calculations are shown. (For details of this calculation, see tables S1 and S2.) 

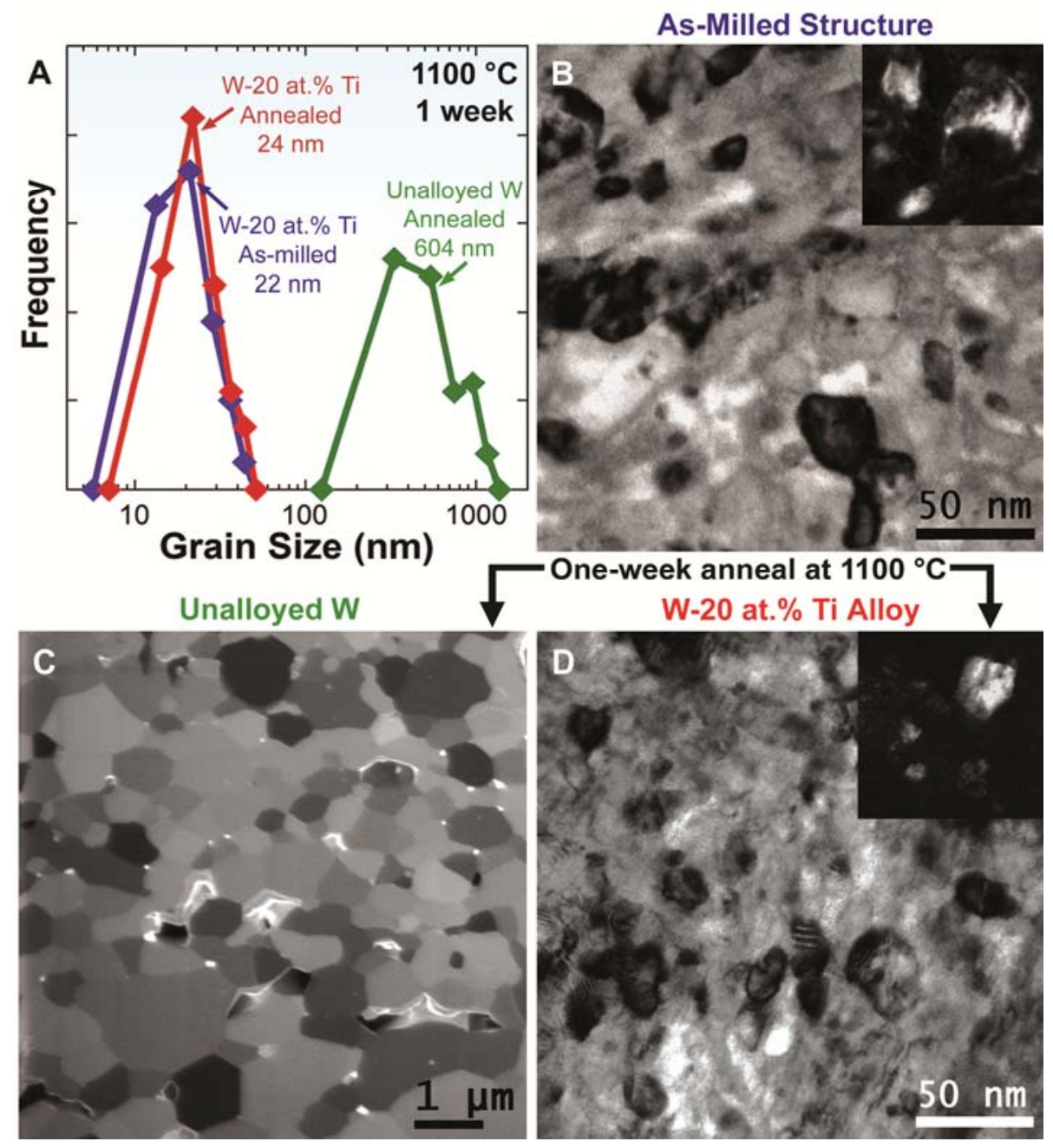

Fig. 2. Pre- and postannealing grain structures of tungsten powders after one week at $1100^{\circ} \mathrm{C}$. (A) The grain size histograms reveal only a minor change in the W-20 atomic \% Ti alloy after prolonged annealing and an almost two-orders-of-magnitude coarsening in unalloyed W. (B) The bright-field transmission electron microscopy (TEM) image shows a uniform distribution of nanometer-sized grains in the as-milled structure of the W-20 atomic \% Ti alloy, with the darkfield TEM image (inset) showing different diffracting crystallites. The postannealing structures vary with alloying: (C) a coarsened grain structure in unalloyed $\mathrm{W}$, presented in a focused ion beam image, and (D) a retained nanocrystalline structure in $\mathrm{W}-20$ atomic \% $\mathrm{Ti}$, shown in a bright-field TEM image with a dark-field TEM (inset). 


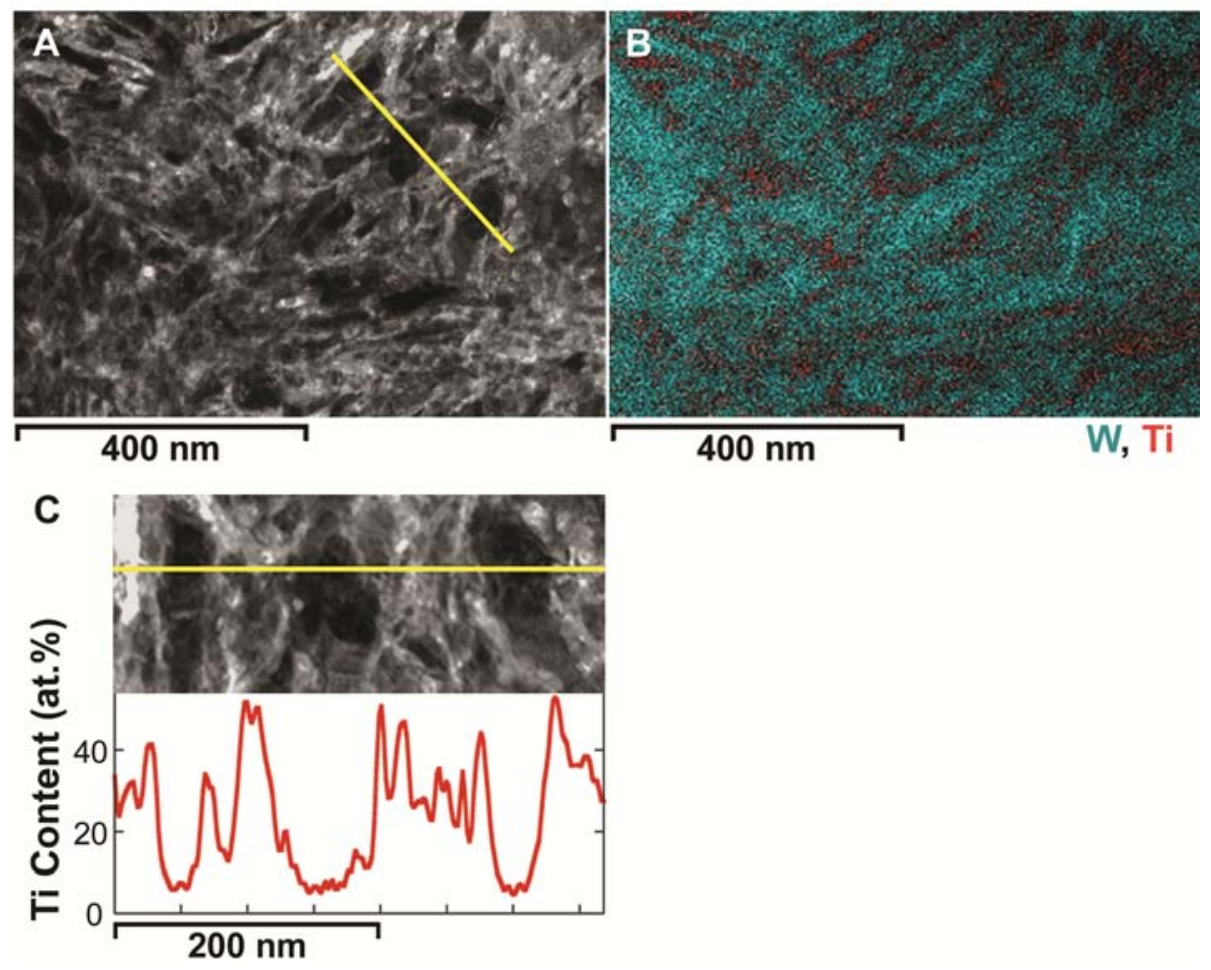

Fig. 3. Chemical analysis of the annealed W-20 atomic \% Ti alloy. (A) Scanning transmission electron microscopy imaging shows the darker contrast from a heavier element and the brighter contrast from a lighter element. (B) The W-Ti elemental map confirms a nanoscale heterogeneous structure instead of a more homogeneous solute distribution expected from bulk thermodynamics. (C) The Ti compositional line scan across the three larger W-rich regions indicated by the yellow line in (A) further illustrates Ti atoms being depleted at the W-rich grain centers. The entire volume is apparently a polycrystalline BCC structure, although with nanoscale composition gradients. 